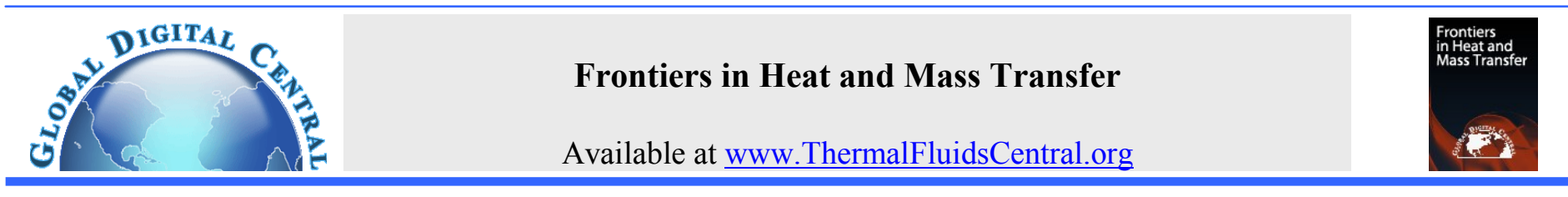

\title{
UREA-WATER DROPLET PHASE CHANGE AND REACTION MODELLING: MULTI-COMPONENT EVAPORATION APPROACH
}

\author{
Viraj S. Shirodkar \\ Citec Engineering India Pvt. Ltd., Pune, Maharashtra, 411057, India
}

\begin{abstract}
Urea-water solution droplet evaporation is modelled using multi-component droplet evaporation approach. The heat and mass transfer process of a multi-component droplet is implemented in the Langrangian framework through a custom code in ANSYS-Fluent R15. The evaporation process is defined by a convection-diffusion controlled model which includes the effect of Stefan flow. A rapid mixing model assumption is used for the droplet internal physics. The code is tested on a single multi-component droplet and the predicted evaporation rates at different ambient temperatures are compared with the experimental data in the literature. The approach is used to model the injection of urea-water solution spray in a duct carrying hot air to predict the urea to ammonia conversion efficiency. Thermolysis reaction of the evaporated urea and the hydrolysis of the byproduct iso-cyanic acid are solved as volumetric reactions in the Eulerian framework using laminar finite rate approach. The spray simulation results are compared with the experimental data and the numerical results of surface reaction based direct thermolysis approach available in the literature.
\end{abstract}

Keywords: Urea Injection, Droplet Mass Transfer, Stefan Flow, Thermolysis, Hydrolysis;

\section{INTRODUCTION}

One of the methods used for exhaust after-treatment in diesel engines is the use of urea-water solution spray in the exhaust gases. The main idea is to introduce ammonia in the exhaust gases entering a catalytic converter. The ammonia acts as a reducing agent in the catalytic converter to reduce the NOx. Urea-water solution is used as the source of ammonia. Ideally urea should be fully converted into ammonia before entering the catalytic converter section. In practice any unconverted urea remains as deposits in the duct and should be avoided. In the numerical modelling of this process, the physics that needs to be addressed involve droplet heating, evaporation, thermolysis and hydrolysis reactions. The aim of the current study is to validate a convection-diffusion controlled multi-component evaporation approach on a single droplet of urea-water solution and apply it for a droplet spray simulation coupled with volumetric thermolysis and hydrolysis reactions to predict the ammonia conversion efficiency.

\subsection{Droplet Heating \& Water Evaporation}

The initial heating of urea-water solution droplet causes temperature rise and evaporation of water. This leads to change in the concentration and properties of a droplet as it continues losing water. In such a multicomponent droplet, heat and mass transfer can be modelled in different ways as discussed in the literature (Birkhold et al., 2007; Sazhin, 2006). The simplest approach assumes constant temperature, species concentration and hence the dependent properties throughout the droplet at a given instant of time. This is known as the rapid mixing model. Other approaches like the diffusion limit model consider temperature and species concentration gradients by inclusion of mass and energy diffusion in a droplet. The effective diffusion model includes flow circulation in a droplet through some empirical correlations. More complex models describe the internal circulation through vortex dynamics models. The study of Birkhold et al. (2007) shows that a rapid mixing model is sufficient and computationally cheap for urea injection studies with no significant improvement using the diffusion limit model. Abu-Ramadan et al. (2011) report that the deviation between the rapid mixing and the diffusion limit models is significant only at lower temperatures and for large droplet sizes. Ryddner and Trujillo (2015) suggest that in case of water entrapment inside the shell of precipitated urea, the diffusion limit model is more appropriate to capture the spatial variation in the concentration of urea inside a droplet.

\subsection{Mass Transfer across a Droplet}

The mass transfer across a droplet can be modelled by two methods depending on the physics of the problem. If the expected mass transfer across the droplet is slow without any dominant convective mass exchange with the surrounding, a diffusion controlled model can be used. The species transport is diffusion dominant and the mass transfer rate follows the Fick's Law given by Eq (1).

$$
\frac{d m_{d, i}}{d t}=-\pi D_{d} \Gamma_{i-\infty} S h_{\infty} M W_{i}\left(\chi_{s, i}-\chi_{\infty, i}\right)
$$

In an exhaust duct, the temperature difference between a droplet and the surroundings is high. In this scenario the above approach defined by Fick's law is not sufficient to capture the correct physics. The evaporated species at the interface displaces the gaseous fluid in the surrounding region generating a mean flow at the interface. The convective transport of species across a droplet or Stefan flow plays an important role in addition to the diffusive transport. Hence in such cases a convection-diffusion controlled model is used. The mass transfer phenomenon in this approach is given by Eq. (2).

$\frac{d m_{d, i}}{d t}=-\pi D_{d} \rho_{\infty, f} \Gamma_{i-\infty} S h_{\infty} \ln \left(1+B_{M, i}\right)$ 


\subsection{Urea Depletion, Thermolysis \& Hydrolysis}

The experimental observation gives some insight into the physics that governs the conversion of multi-component droplet of urea-water solution to gaseous ammonia in hot surrounding conditions. As the droplet is heated it absorbs heat and initially releases water content. Melting of urea occurs at around $406 \mathrm{~K}$ as reported by Sangster (1999). Schaber et al. (2004) reports the vaporization of molten urea at $413 \mathrm{~K}$ and direct decomposition into ammonia and isocyanic acid at temperatures above $425 \mathrm{~K}$. The experiments on a single droplet by Wang et al. (2009) also show fast depletion of urea at temperatures above $423 \mathrm{~K}$ conforming to the observations of Schaber et al. (2004). Whereas the experimental data of Emel'Yanenko et al. (2006) and Bernhard et al. (2011) suggests urea phase change to gaseous state at temperatures around $406 \mathrm{~K}$. Different numerical approaches have been used to solve this problem. The numerical results of Kim et al. (2004) using wet combustion model in Fluent show good match with the overall experimental data for droplet spray. In this approach the diffusion controlled evaporation of water component of a droplet is modelled followed by solid urea devolatilization. The gaseous urea then undergoes thermolysis reaction to produce isocyanic acid and carbon dioxide. In addition, hydrolysis reaction occurs between the isocyanic acid formed during urea thermolysis and the water content in the surrounding medium to give ammonia and carbon dioxide. Kim et al. (2004) treats these reactions in the Eulerian framework. A direct thermolysis study by Birkhold et al. (2007) using CFD code Fire, also shows a reasonable match with the experiments. The approach uses convection-diffusion controlled model for the evaporation of water component and a kinetic model for the direct conversion of urea (solid or molten state) into ammonia and isocyanic acid through thermolysis reaction at the droplet surface. Both these approaches require data on the kinetic parameters for the droplet urea devolatilization or the direct thermolysis of urea. These parameters have to be arrived at using experimental data. Lundstörm et al. (2011) point out the discrepancy in such approaches due to the prediction of unrealistic urea temperatures. Alternative to direct thermolysis is multi-component vaporization approach. Abu-Ramadan et al. (2011) conducted studies on a single droplet of urea-water solution, using direct thermolysis and multicomponent vaporization approaches. The comparison shows better suitability of vaporization approach over direct thermolysis in terms of droplet depletion rates and droplet temperatures. In the vaporization approach the critical data is the urea saturation vapor pressure variation with temperature. In this approach the initiation of urea evaporation occurs before the complete depletion of water content. ClasiusClayperon equation and the experimental data of Emel'Yanenko et al. (2006) are used by Abu-Ramadan et al. (2011) to analytically calculate the saturation vapor pressure. The first law of thermodynamics is used to derive the urea latent heat of vaporization variation with temperature, required in the calculation of saturation pressure. The current study uses convection-diffusion controlled multi-component droplet evaporation approach for urea-water solution droplet and spray simulation using a rapid mixing model followed by volumetric reactions in the Eulerian framework. An attempt is made to check the suitability of such an approach, fine tune the required inputs and the formulation and validate with the experimental data for a single droplet and spray evaporation.

\section{NUMERICAL APPROACH FOR THE STUDY}

Steady state numerical simulations are conducted for the evaporation of a single droplet and a spray of urea-water solution in the EulerianLagrangian framework of ANSYS-Fluent R15. Two-way interactions are allowed between the Lagrangian and the Eulerian phases. The heat and mass equations of the Lagrangian particle are solved in a coupled manner. The model for discrete particle heat and mass transfer of a multi-component droplet is implemented through a user defined $\mathrm{C}$ code assuming a rapid mixing model for the liquid droplet internal physics and a convection-diffusion controlled droplet heat and mass exchange. The urea thermolysis and hydrolysis reactions are modelled as volumetric reactions in the gaseous phase using laminar finite-rate interactions between turbulence and chemistry. Second order upwind scheme is used for discretization in momentum, turbulence and species transport equations. Fluent uses lower order implicit and higher order Runge-Kutta schemes for particle tracking depending on the stability. Spherical shape is assumed for the droplets with the inbuilt drag law given by Morsi \& Alexander (1972). Radiative heat transfer is not considered in the study. The residual convergence criterion of $1 \mathrm{e}-3$ is used for continuity momentum \& turbulence equations and $1 \mathrm{e}-6$ for species \& energy equations. The droplet impingement on the duct wall is not expected based on the experimental flow visualization results of Kim et al. (2004). Any stray droplet impingement is simplified as particle reflection without deposition or film formation at the wall.

\subsection{Heat and Mass Exchange for Multi-Component Droplet}

The heat and mass exchange occurs at the surface of a droplet and requires the calculation of quantities like mole and mass fractions at the surface. In the current study Raoult's law is used to calculate these quantities. The mole and mass fractions of urea and water at the droplet surface are expressed by Eqs. (3) and (4) respectively.

$$
\begin{gathered}
X_{i, s}=X_{i, l} \frac{p_{s a t, i}}{p_{\infty}} \\
Y_{i, s}=\frac{X_{i, s} M W_{i}}{\sum_{j=1}^{N} X_{j, s} M W_{j}}
\end{gathered}
$$

Equation (5) shows the saturation pressure curve for water given by Tetens (1930). From the experimental data, Birkhold (2006) estimates the urea saturation curve as in Eq. (6).

$$
\begin{aligned}
& p_{\text {sat }, \text { water }}=610.78 e^{17.2694\left(T_{l}-273.16\right) /\left(T_{l}-35.86\right)} \\
& p_{\text {sat, urea }}=e^{29.9548-\left(10876.1 / T_{l}\right)}
\end{aligned}
$$

The study uses a rapid mixing model for the liquid droplet calculations. The urea mass concentration of a droplet varies at the time rate given by Eq. (7). The convection-diffusion controlled mass exchange is modelled at the interface. The rate of mass transfer when the total vapor pressure is less than the computational cell pressure is given by Eq. (8). Sherwood number is calculated using Ranz-Marshall correlation. The binary diffusivity is calculated between the evaporating species and the surrounding mixture approximated as air. The calculation is based on Leonard-Jones potentials. The quadratic curves fitted through the calculated diffusivity data are given in Appendix A.

$$
\begin{aligned}
\frac{d Y_{u, l}}{d t} & =-\frac{\left(d m_{v a p} / d t\right)}{m_{d}} Y_{u, l} \\
\frac{d m_{d, i}}{d t} & =-\phi_{i} \pi D_{d} \rho_{m i x, f} \Gamma_{i-m i x, f} S h_{m i x, f} \ln \left(1+B_{M, i}\right) \\
B_{M, i} & =\frac{Y_{i, f}-Y_{i, \infty}}{1-Y_{i, f}} \\
S h_{\text {mix }, f} & =\left(2.0+0.552 \operatorname{Re}_{d, m i x, f}^{1 / 2} S c_{d, m i x, f}^{1 / 3}\right)
\end{aligned}
$$

The thermo-physical fluid properties for the heat and mass transfer and the mass fraction of an evaporating species in the film are calculated at a mean film temperature using $1 / 3$ rd rule. $T_{s}=T_{l}$ is used in calculations as per the rapid mixing model.

$$
\begin{aligned}
& T_{f}=T_{s}+\frac{1}{3}\left(T_{s}-T_{\infty}\right) \\
& Y_{i, f}=Y_{i, s}+\frac{1}{3}\left(Y_{i, s}-Y_{i, \infty}\right)
\end{aligned}
$$


The energy balance of a droplet is given Eq. (14). The heat added from the surroundings by convection is summed up as the droplet sensible heat and the latent heat required for vaporization. To take into account Stefan flow effects, the convective heat transfer is modified by Spalding heat transfer number. Some studies assume Spalding numbers for mass transfer and heat transfer to be equal. But this assumption is a special case as described by Sazhin (2006). Birkhold et al. (2007) express the relation between Spalding heat and mass transfer numbers as given by Eq. (15). For sensible heating, Birkhold et al. (2007) suggests adding the latent heat of melting $(14.5 \mathrm{~kJ} / \mathrm{mol})$ to the latent heat of vaporization $(87.1 \mathrm{~kJ} / \mathrm{mol})$ for the urea at standard temperature $T_{\text {std }}=298 \mathrm{~K}$. The latent heat at the mean film temperature is calculated by Eq. (17) using the first law of thermodynamics as suggested by AbuRamadan et al. (2011).

$$
\begin{aligned}
& Q_{\text {convection }}=Q_{\text {sensibleheat }}+Q_{\text {latent-heat }} \\
& \sum \phi_{i} \pi D_{d} k_{\text {mix }, f} N u_{\text {mix }, f} \frac{\ln \left(1+B_{T, e, i}\right)}{B_{T, e, i}}\left(T_{\infty}-T_{s}\right)= \\
& B_{T, e, i} C p_{l} \frac{d T}{d t}+\sum \frac{d m_{\text {vap }, i}}{d t} L_{\text {vap }, i} \\
& x_{i}=\left(\frac{S h_{\text {mix }, f}}{N u_{\text {mix }, f}}\right)\left(\frac{C p_{\text {vap }, i, f}}{C p_{\text {mix }, f}}\right)\left(\frac{1}{L e_{\text {mix }, f}}\right) \\
& L_{\text {vap }, i}=L_{\text {vap }, \text { std }, i}+\int_{T_{s t d}}^{T_{s}} C p_{\text {vap }, i} d T-\int_{T_{s t d}}^{T_{s}} C p_{l, i} d T
\end{aligned}
$$

In a multi-component droplet, if the total vapor pressure of the droplet exceeds the computational cell pressure then the boiling law is applied. The mass transfer rate for the boiling law is given by Eq. (19). Ranz-Marshall correlation is used for calculating Nusselt number.

$p_{t o t}=\sum X_{i, l} p_{s a t, i}$

$\frac{d m_{d, i}}{d t}=-\phi_{i} \pi D_{d} \frac{k_{\text {mix }, f}}{C p_{\text {vap }, i, f}} N u_{\text {mix }, f} \ln \left(1+B_{T, b, i}\right)$

$N u_{m i x, f}=\left(2.0+0.552 \operatorname{Re}_{d, m i x, f}^{1 / 2} \operatorname{Pr}_{d, m i x, f}^{1 / 3}\right)$

$B_{T, b, i}=\frac{C p_{\text {vap }, i, f}\left(T_{\infty}-T_{s}\right)}{L_{\text {vap }, i}}$

\subsection{Thermolysis \& Hydrolysis}

Thermolysis of the evaporated urea is modelled as a volumetric reaction in the gaseous phase. Gaseous urea decomposes into ammonia gas and isocyanic acid vapors as in Eq. (22). The activation energy constant for this reaction is used from the study of Kim et al. (2004). The preexponent is obtained by trial and error to match reasonably with the experimental data of Kim et al. (2004). The enthalpy change for this reaction given by Birkhold et al. (2007) is $95 \mathrm{~kJ} / \mathrm{mol}$.

$\left(\mathrm{NH}_{2}\right)_{2} \mathrm{CO}=\mathrm{NH}_{3}+\mathrm{HNCO}$ $\frac{d c_{\text {th }}}{d t}=\chi_{\text {urea }} A_{t h} e^{\left(-E_{t h} / R T_{\infty}\right)}$

$A_{t h}=940 \mathrm{~s}^{-1}, E_{t h}=2.94 \times 10^{7} \mathrm{~J} / \mathrm{kmol}$

Hydrolysis reaction, wherein isocyanic acid reacts with water vapor to form ammonia and carbon dioxide, is given by Eq. (25). Yim et al. (2004) gives the rate constants for the hydrolysis reaction. These rate constants are the same as used in the study by Birkhold (2007). The enthalpy change for this reaction given by Koebel et al. (2000) is 96 $\mathrm{kJ} / \mathrm{mol}$.

$$
\begin{aligned}
& \mathrm{HNCO}+\mathrm{H}_{2} \mathrm{O}=\mathrm{NH}_{3}+\mathrm{CO}_{2} \\
& \frac{d c_{h y}}{d t}=\chi_{H N C O} A_{h y} e^{\left(-E_{h y} / R T_{\infty}\right)} \\
& A_{t h}=2.5 \times 10^{5} \mathrm{~s}^{-1}, E_{t h}=6.22 \times 10^{7} \mathrm{~J} / \mathrm{kmol}
\end{aligned}
$$

\section{VALIDATION PROBLEMS}

The problems validated in the study include single multi-component droplet evaporation and a spray of multi-component droplets with a size distribution simulated for evaporation, thermolysis and hydrolysis. The results are compared with the experimental data in the open literature. The computational domain used for both the validation problems is an adiabatic duct of diameter $300 \mathrm{~mm}$ and a length of $6.5 \mathrm{~m}$ as shown in Fig. 1. The initial droplet temperature is assumed to be $300 \mathrm{~K}$. Lagrangian physics dominates this problem and hence a wall function type of uniform hexahedral mesh with a cell count of 68000 is used in the study. The average $\mathrm{y}^{+}$at the wall is maintained approximately around 60 with an aspect ratio of 2. No significant change in the species concentration is observed in the Eulerian domain with further mesh refinement.

\subsection{Single Multi-Component Droplet}

Experimental data on evaporation time and droplet size variation during evaporation of a single droplet of urea-water solution is given by Wang et al. (2009). A solution of $32.5 \%$ urea by weight is used in the study. The combinations of droplet size and ambient conditions used in the current numerical study are listed in the Table 1 . The corresponding evaporation rates and droplet diameter variation with the time is predicted and compared with the experimental data. The ambient air is considered stagnant. No turbulence model is used in the simulation. Thermolysis and hydrolysis reactions are neglected for the single droplet evaporation.

Table 1 Droplet size and ambient conditions for droplet evaporation

\begin{tabular}{|c|c|}
\hline Droplet Diameter $(\mathrm{mm})$ & Ambient Temperature $(\mathrm{K})$ \\
\hline 0.92 & 573 \\
\hline 0.865 & 623 \\
\hline 0.815 & 673 \\
\hline
\end{tabular}

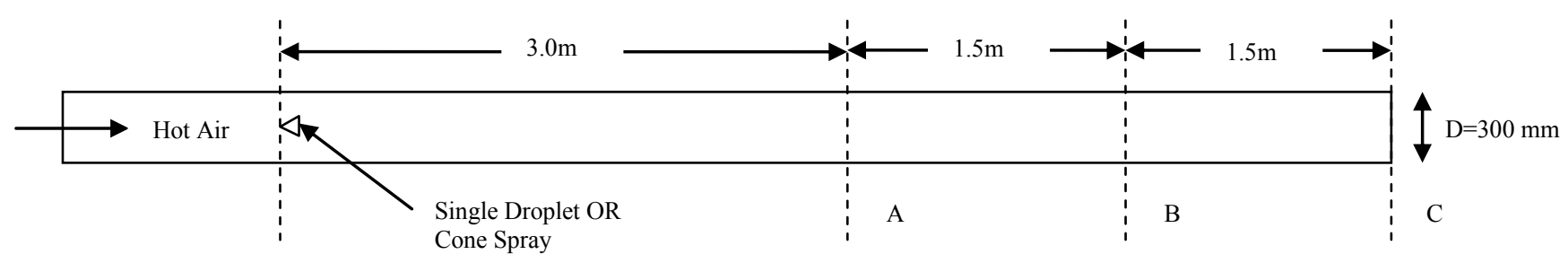

Fig. 1 Problem Domain 


\subsection{Spray of Multi-Component Droplets}

Urea-water solution spray experiments are conducted by Kim et al. (2004). The hot air is introduced in the duct at different velocities and temperatures as shown in Table 2. Urea-water solution of $40 \%$ by weight of urea is injected into the duct at a mass flow rate of $3.3 \mathrm{e}-4$ $\mathrm{kg} / \mathrm{s}$. The initial velocity of the spray particles is $10.6 \mathrm{~m} / \mathrm{s}$. The spray is modelled as a hollow cone of 6 droplet streams with Rosin-Rammler distribution. The mean distribution size of 44 microns and a spread of 3.27 are used for the spray characteristics. The droplet size variation limits are assumed to be 5-200 microns with 10 diameter bands. An initial simulation is conducted for inlet air velocity of $5.4 \mathrm{~m} / \mathrm{s}$ to check the droplet spray width. Eulerian phase turbulence is modelled by realizable k-epsilon model with wall function mesh. Fully developed air velocity and turbulence profiles are used at the duct inlet. The particle dispersion due to turbulence is not considered in the study.

Table 2 Surrounding air temperature and velocity for spray simulation

\begin{tabular}{|c|c|}
\hline Air Temperature $(\mathrm{K})$ & Air Velocity $(\mathrm{m} / \mathrm{s})$ \\
\hline 573 & 9.1 \\
\hline 623 & 9.0 \\
\hline 673 & 8.3 \\
\hline
\end{tabular}

\section{RESULTS \& DISCUSSION}

The temporal size variation during the evaporation of a single droplet of urea-water solution is compared with the experimental data of Wang et al. (2009). The squared diameter of droplet is normalized by the square of initial droplet diameter and the variation with respect to the normalized time is plotted in Fig.2. The observed droplet evaporation rate is higher in the initial phase in the presence of water content. After the water is exhausted the evaporation rate drops and is now governed by the urea saturation pressure. This drop in the evaporation rate after the depletion of water content is significant at a lower temperature of $573 \mathrm{~K}$. Droplet diameter variation curve matches well for the higher temperatures of $623 \mathrm{~K}$ and $673 \mathrm{~K}$ in the initial phase. As the droplet water content is reduced, a deviation is observed between the experimental and the numerical results. This is due to the uncertainty in the urea saturation pressure curve. The initial phase deviation between the predicted values and the experiments is high for the lower temperature of $573 \mathrm{~K}$. At this temperature, the experimental and the numerical evaporation curves follow parallel to each other after the water depletion. This suggests that at a lower temperature of $573 \mathrm{~K}$, the rapid mixing model may not be valid. At such temperatures, the internal temperature gradient effects on the droplet species concentration can play an important role in determining the droplet evaporation rates.

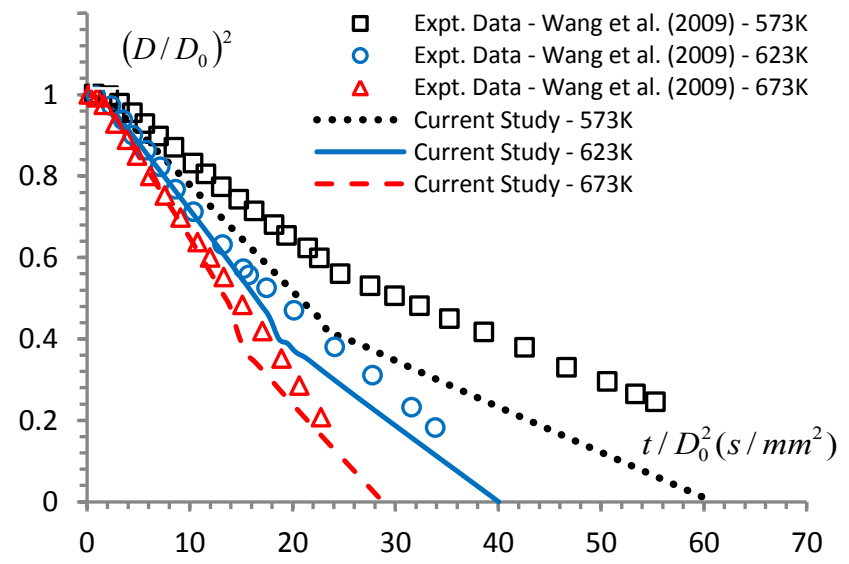

Fig. 2 Non-dimensionalized diameter square variation with time
Figure 3 shows the time variation of droplet temperature at an ambient condition of $623 \mathrm{~K}$. An initial rate of rise in the droplet temperature is steep and decreases near the boiling point of water. Once the water is fully evaporated, the droplet temperature rises steeply and then stabilizes at around $520 \mathrm{~K}$. An evaporating droplet mass variation with time at an ambient temperature of $623 \mathrm{~K}$, with and without the assumption of $B_{M}=B_{T}$ is shown in Fig. 4. The comparison shows a relatively lower instantaneous droplet mass in case of equality assumption. The droplet spray visualization for the inlet air velocity of $5.4 \mathrm{~m} / \mathrm{s}$ and a temperature of $623 \mathrm{~K}$ is shown in Fig. 5 .

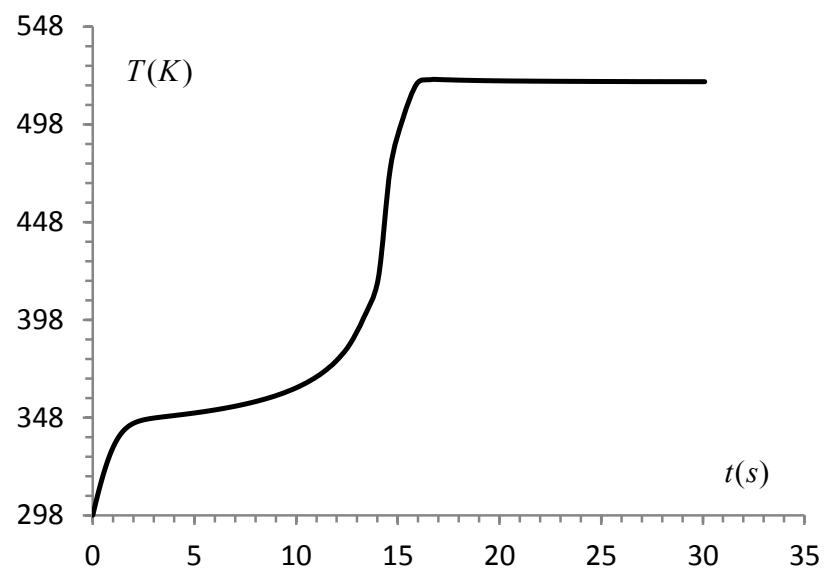

Fig. 3 Droplet temperature variation with time

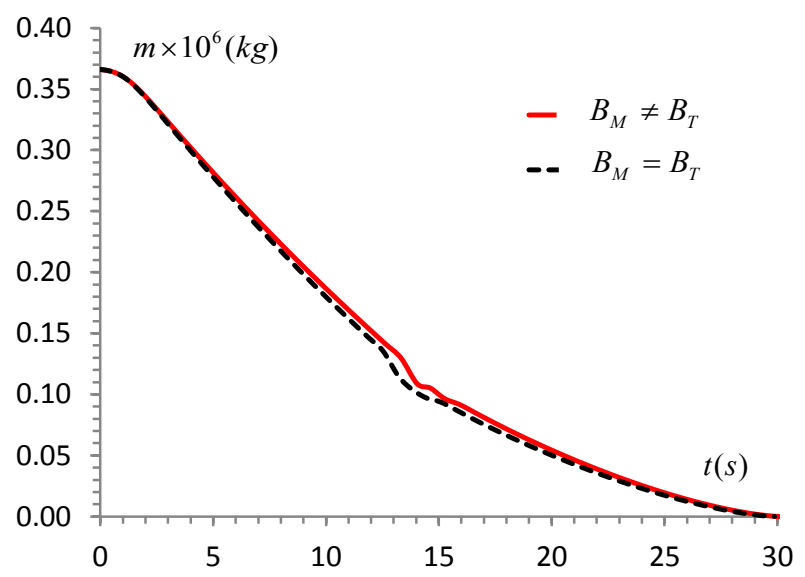

Fig. 4 Effect of Spalding number calculations on evaporation

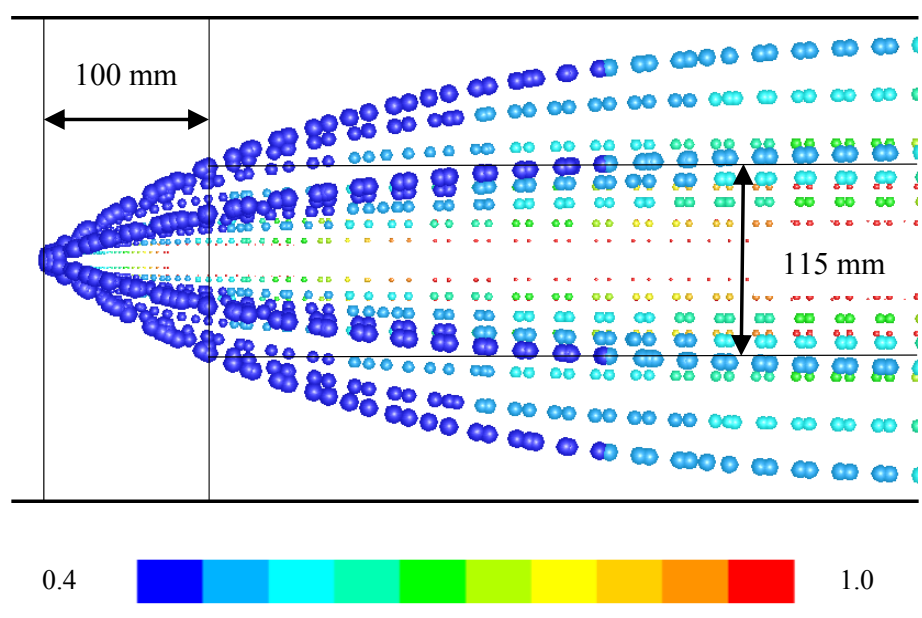

Fig. 5 Droplets coloured by urea mass fraction 
The predicted width of spray at $100 \mathrm{~mm}$ from the injection is $115 \mathrm{~mm}$ and matches closely with the experimentally reported value of $120 \mathrm{~mm}$ by Kim et al. (2004). Lagrangian particle tracks show the smaller droplets traversing shorter paths before completely losing their water content. The simulations conducted based on the parameters in Table 2 predict complete evaporation of the spray droplets for the inlet air temperatures of $623 \mathrm{~K}$ and $673 \mathrm{~K}$. In case of inlet air temperature of 573 $\mathrm{K}$, some droplets are partially evaporated and they pass the duct outlet as smaller droplets of $100 \%$ urea content. The particle residence time increases with the decreasing ambient temperature. This shows the significance of the exhaust duct length and the surrounding fluid temperature for the complete evaporation of urea. The ammonia conversion efficiency is defined as the ratio of the actual ammonia formed in the duct to the ideal possible ammonia conversion from the injected amount of urea. The efficiency versus time plots for the ambient conditions of $573 \mathrm{~K}, 623 \mathrm{~K}$ and $673 \mathrm{~K}$ are shown in Fig.6, Fig. 7 and Fig. 8 respectively. The ammonia content is calculated at the transverse planes A, B and C shown in the Fig.1.

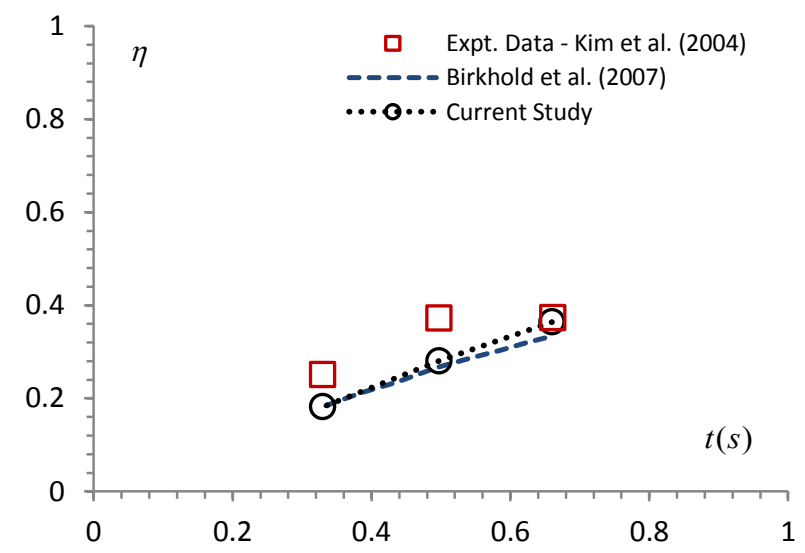

Fig. 6 Ammonia conversion efficiency for 573

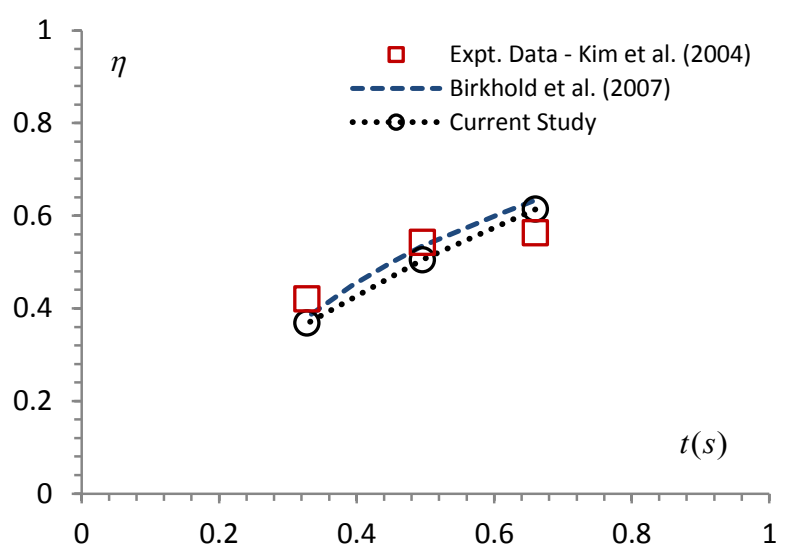

Fig. 7 Ammonia conversion efficiency for $623 \mathrm{~K}$

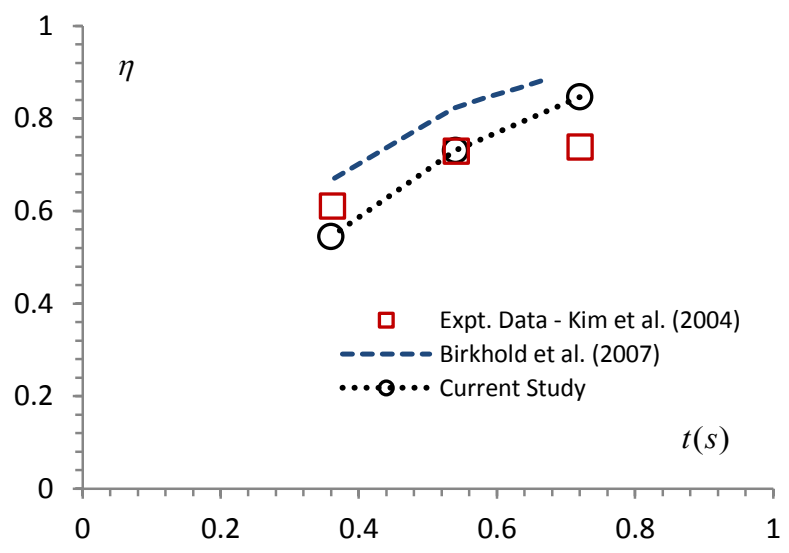

Fig. 8 Ammonia conversion efficiency for $673 \mathrm{~K}$

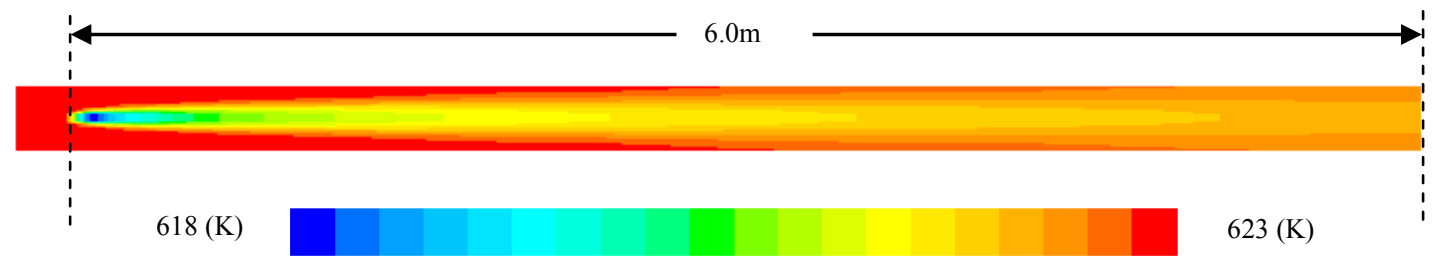

Fig. 9 Temperature distribution in the duct in for ambient air of $623 \mathrm{~K}$

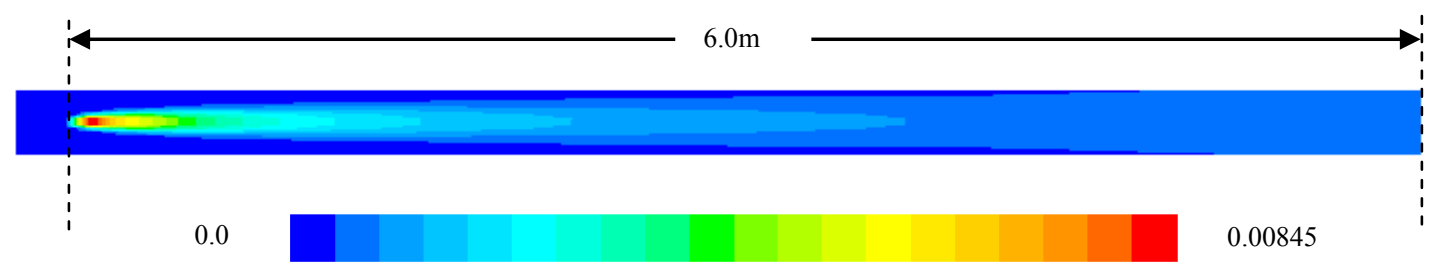

Fig. 10 Mass fraction of water-vapor in the duct for ambient air of $623 \mathrm{~K}$

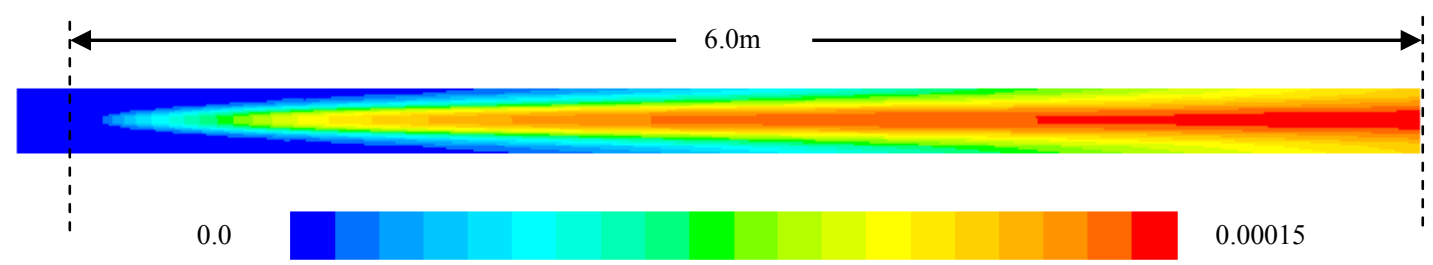

Fig. 11 Mass fraction of ammonia in the duct for ambient air of $623 \mathrm{~K}$ 


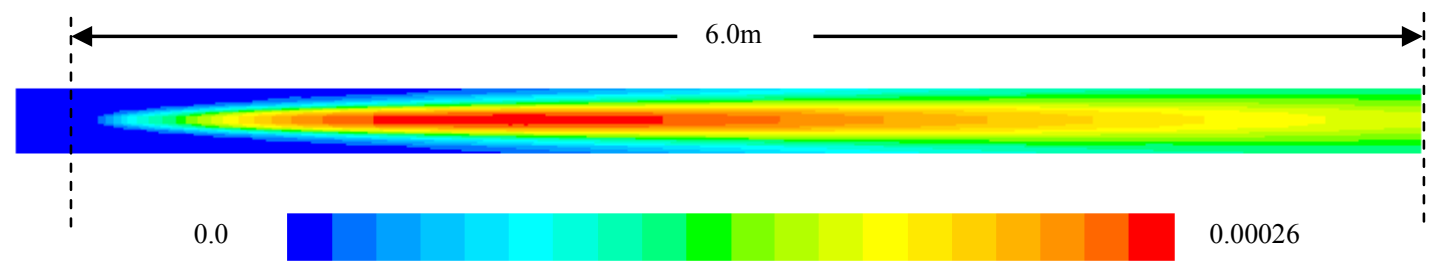

Fig. 12 Mass fraction of iso-cyanic acid in the duct for ambient air of $623 \mathrm{~K}$

A reasonable match is observed with the experimental data of Kim et al. (2004). The experimental data shows that the initial increase in the ammonia conversion efficiency along the duct drops in the downstream direction, especially at high temperatures. This effect is captured to some extent in the numerical results of $673 \mathrm{~K}$. As the droplet sizes used in case of spray are much smaller than the single droplet simulation, the spatial variation effects due to finite diffusivity at lower temperature are comparatively less critical. A deviation is observed at a higher temperature of $673 \mathrm{~K}$ and would require accurate reaction kinetics data for improved predictions. Figure 9 shows the duct temperature distribution in the Eulerian domain. A local temperature drop is seen in the injection region due to the evaporation of predominantly water in the initial narrow cone of the spray as shown in Fig. 10. As the droplets disperse in the downstream direction, the temperature change in the duct is more uniform. In addition, the endothermic thermolysis reaction also causes temperature drop in the hot air. In the direct thermolysis approach this heat is extracted from the surroundings at the droplet surface whereas in vaporization approach this heat is extracted over a spread out region due to the convection of evaporated urea vapor. The exothermic hydrolysis reaction is more active in the downstream region. This region is identified by the increased ammonia as shown in Fig. 11 or the depleted iso-cyanic acid in Fig 12. The ammonia contours in Fig. 11 show that thermolysis reaction is active at a location downstream of the injection point. This is due to the time lag in the initiation of urea evaporation.

\section{CONCLUSIONS}

A multi-component droplet heat and mass transfer model is implemented based on convection-diffusion controlled evaporation and a rapid mixing model assumption for the droplet internal characteristics. The parameters like droplet evaporation time, droplet diameter variation, conversion of urea to ammonia in a spray simulation are compared with the experimental data. The study shows promising results for the simulations conducted on a single droplet and spray of urea-water solution. The lack of clarity in the exact physical nature of urea phase change is a major factor of uncertainty. There is a need for fine-tuning the saturation pressure curve of urea with the help of experimental data. Another factor which can add to the uncertainty is the diffusion coefficient between the evaporating species and the surrounding medium. The current study uses theoretical formulation based on Leonard-Jones potentials for the calculation of binary diffusivity. The droplet physics related to internal flow circulation, the spatial concentration variation and the radiative heat transfer is not considered in this study. A deviation from the experimental data is observed in the single droplet evaporation rate at a lower temperature of $573 \mathrm{~K}$. The droplet internal gradients and flow circulation plays important role at low temperatures and hence need diffusion limit model to account for this. The study shows that the assumption in the calculation of Spalding number has an effect on the calculation of evaporation rate. In case of urea-water solution spray, the approach of multi-component droplet evaporation with volumetric gas phase reactions show predictions comparable to the direct thermolysis approach. The predicted droplet spray width and the ammonia conversion efficiency match the experimental data of Kim et al. (2004) with a reasonable accuracy. There is a scope for refinement in the representation of thermolysis and hydrolysis reaction kinetics in order to improve the prediction of variation in ammonia formation along the duct.

\section{NOMENCLATURE}

$A \quad$ pre-exponent factor for reaction

$B_{M} \quad$ Spalding number for Mass Transfer

$B_{T} \quad$ Spalding number for Energy transfer

$D \quad$ droplet diameter (m)

$C p \quad$ specific heat at constant pressure $(\mathrm{J} / \mathrm{kgK})$

$E \quad$ activation energy $(\mathrm{J} / \mathrm{kmol})$

$k \quad$ thermal conductivity $(\mathrm{W} / \mathrm{mK})$

$L \quad$ latent heat $(\mathrm{J} / \mathrm{kg})$

Le Lewis number

$m \quad$ mass $(\mathrm{kg})$

$M W \quad$ molecular weight $(\mathrm{kg} / \mathrm{kmol})$

$\mathrm{Nu} \quad$ Nusselt number

$\mathrm{Pr} \quad$ Prandtl number

$p \quad$ pressure $(\mathrm{Pa})$

Re Reynolds number

$Q \quad$ heat rate (W)

Sc Schmidt number

Sh Sherwood number

$T$ temperature (K)

$t \quad$ time (sec)

$X \quad$ mole fraction

$x \quad$ exponent factor for Spalding number

$Y \quad$ mass fraction

\section{Greek Symbols}

$\Gamma \quad$ diffusivity (m2/s)

$\chi \quad$ molar concentration $(\mathrm{kmol} / \mathrm{m} 3)$

$\rho \quad$ fluid density $(\mathrm{kg} / \mathrm{m} 3)$

$\phi \quad$ volume fraction

$\eta \quad$ ammonia conversion efficiency

\section{Subscripts}

$B \quad$ interface of gas and liquid

d droplet

$f \quad$ film

hy hydrolysis

$i$ component

$l \quad$ liquid phase

mix mixture of gases

$s \quad$ droplet surface

sat saturation condition

std standard condition (298K)

th thermolysis

vap vapor phase

0 initial condition

$\infty \quad$ ambient conditions 


\section{APPENDIX A: PHYSICAL PROPERTY DATA}

The section gives data and information on calculation approach for some of the important physical properties of the different gases and liquids used in the study.

\section{Droplet Component Properties}

A constant density assumption is used for urea $(1270 \mathrm{~kg} / \mathrm{m} 3)$ and water $(998 \mathrm{~kg} / \mathrm{m} 3)$ in the liquid state. The binary diffusivity of evaporating gas at the droplet surface into the surrounding mixture is analytically calculated as described by Nellis and Klein (2008). Leonard-Jones length and energy parameters are used from the ANSYS-Fluent material database. Quadratic equations fitted through the calculated data are given in Eq. (A1) and Eq. (A2). The binary diffusivity is in $\mathrm{m} 2 / \mathrm{s}$ and the calculations are performed for the mean film in $\mathrm{K}$.

$$
\begin{aligned}
& \Gamma_{\text {urea-air }}=8.206 \times 10^{-11} T^{2}+3.143 \times 10^{-8} T-4.509 \times 10^{-6} \\
& \Gamma_{\text {water-air }}=1.625 \times 10^{-10} T^{2}+5.864 \times 10^{-8} T-8.630 \times 10^{-6}
\end{aligned}
$$

The specific heat capacity relation given by Kontin et al. (2010) is used for the urea component in droplet. A fourth-order polynomial equation is fitted through the specific heat capacity data of water available in open literature. $\mathrm{Cp}$ is in $\mathrm{J} / \mathrm{kgK}$ and $\mathrm{T}$ is in $\mathrm{K}$ in Eq. (A3) and Eq. (A4).

$$
\begin{aligned}
C p_{l, \text { urea }}= & 4.1147 T-291.06 \\
C p_{l, \text { water }}= & 8.802 \times 10^{-7} T^{4}-1.233 \times 10^{-3} T^{3}+6.552 \times 10^{-1} T^{2} \\
& -1.557 \times 10^{2} T+1.808 \times 10^{4}
\end{aligned}
$$

\section{Droplet Mixture Properties}

The droplet mixture properties such as density and specific heat capacity are calculated using the inbuilt mixing law in ANSYS-Fluent.

\begin{tabular}{|l|l|}
\hline Density & Volume-Weighted Mixing Law \\
\hline Specific Heat Capacity & Mass Weighted Mixing Law \\
\hline
\end{tabular}

\section{Gas Phase Properties}

The study uses specific heat capacity expression from ANSYS-Fluent material database for the gaseous state. $\mathrm{Cp}$ is in $\mathrm{J} / \mathrm{kgK}$ and $\mathrm{T}$ is in $\mathrm{K}$ in Eq. (A5) and Eq. (A6). Inbuilt kinetic theory based formulation is used for calculation of dynamic viscosity and thermal conductivity of gases.

$$
\begin{aligned}
& C p_{\text {vap, urea }}=-1.156 \times 10^{-9} T^{4}+3.216 \times 10^{-6} T^{3}-2.932 \times 10^{-3} T^{2} \\
& +1.603 \times 10^{0} T+1.563 \times 10^{3} \quad(300<T<1000) \\
& C p_{\text {vap,water }}=1.474 \times 10^{-10} T^{4}-1.760 \times 10^{-7} T^{3}-2.023 \times 10^{-4} T^{2} \\
& +4.018 \times 10^{-1} T+4.093 \times 10^{2} \quad(300<T<1000)
\end{aligned}
$$

\section{Gaseous Mixture Properties}

The gaseous mixture properties are calculated using the inbuilt mixing law and kinetic theory based formulations in ANSYS-Fluent.

\begin{tabular}{|l|l|}
\hline Density & Ideal Incompressible Gas \\
\hline Thermal Conductivity & Mass-Weighted Mixing law \\
\hline Specific Heat Capacity & Mass-Weighted Mixing law \\
\hline Viscosity & Mass-Weighted Mixing law \\
\hline Mass Diffusivity & Kinetic Theory \\
\hline Thermal Diffusivity & Kinetic Theory \\
\hline
\end{tabular}

\section{REFERENCES}

Birkhold, F., Meingast, U., Wassermann, P., Deutschmann, O., 2007, "Modeling and Simulation of the Injection of Urea-Water-Solution for Automotive SCR DeNOx-Systems", Applied Catalysis B: Environmental, 70, 119-127. http://dx.doi.org/10.1016/j.apcatb.2005.12.035

Sazhin, S., 2006, "Advanced Models of Fuel Droplet Heating and Evaporation", Progress in Energy and Combustion Science, 32, 162214.

http://dx.doi.org/10.1016/j.pecs.2005.11.001

Abu-Ramadan, E., Saha, K., Li, X., 2011, "Modeling the Depleting Mechanism of Urea-Water-Solution Droplet for Automotive Selective Catalytic Reduction Systems. " AIChE Journal, 57, 3210-3225.

http://dx.doi.org/10.1002/aic.12523

Ryddner, D., Trujillo, M., 2015, "Modeling Urea-Water Solution Droplet Evaporation", Emission Control Science \& Technology, 1, 8097.

http://dx.doi.org/10.1007/s40825-014-0006-3

Sangster, J., 1999, "Phase Diagrams and Thermodynamic Properties of Binary Systems of Drugs", J. Phys. Chem. Ref. Data, 28, 889. http://dx.doi.org/10.1063/1.556040

Schaber, P., Colson, J., Higgins, S., Thielen, D., Anspach, B., Brauer, J., 2004, "Thermal Decomposition (Pyrolysis) of Urea in an Open Reaction Vessel", Thermochimica Acta, 424, 131-142.

http://dx.doi.org/10.1016/j.tca.2004.05.018

Wang, T., Baek, S., Lee, S., Kang, D., Yeo, G., 2009, “Experimental Investigation on Evaporation of Urea-Water-Solution Droplet for SCR Applications", AIChE Journal, 55 (12), 3267-3276.

http://dx.doi.org/10.1002/aic.11939

Emel'Yanenko, V., Kabo, G., Verevkin, S., 2006, "Measurement and Prediction of Thermochemical Properties: Improved Increments for the Estimation of Enthalpies of Sublimation and Standard Enthalpies of Formation of Alkyl Derivatives of Urea", J. Chem. Eng. Data, 51 (1), 79-87.

http://dx.doi.org/10.1021/je050230z

Bernhard, A., Czekaj, I., Elsener, M., Wokaun, A., Krocher, O., 2011, “ Evaporation of Urea at Atmospheric Pressure", J. Phys. Chem. A, 115 (12), 2581-2589

http://dx.doi.org/10.1021/jp112066m

Kim, J., Ryu, S., Ha, J., 2004, "Numerical Prediction on the Characteristics of Spray-Induced Mixing and Thermal Decomposition of Urea Solution in SCR System", Proceedings of 2004 Fall Technical Conferences of the ASME International Combustion Engine Division, Long Beach, California USA.

http://dx.doi.org/10.1115/ICEF2004-0889

Lundström, A., Waldheim, B., Ström, H., Westerberg, B., 2011, "Modelling of Urea Gas Phase Thermolysis and Theoretical Details on Urea Evaporation", Proceedings of the Institution of Mechanical Engineers Part D: Journal of Automobile Engineering, 225 (10), 13921398 http://dx.doi.org/10.1177/0954407011406048

Morsi, S., Alexander, A., 1972, "An Investigation of Particle Trajectories in Two-Phase Flow Systems", J. Fluid Mech., 55 (2), 193 208.

http://dx.doi.org/10.1017/S0022112072001806

Tetens, O., 1930, "Uber Einige Meteorologische Begriffe", Z Geophys., 6, 297-309.

Birkhold, F., 2006, "Selektive Katalytische Reduktion von Stickoxiden 
in Kraftfahrzeugen: Untersuchung der Einspritzung von Harnstoffwasserlösung”, Dissertation, Universität Stuttgart.

Nellis, G., Klein S., 2008, "Heat Transfer", Cambridge University Press, First edition.

Yim, D., Kim, S., Baik, J., Nam, I., Mok, Y., Lee, J., Cho, B., Oh, S., 2004, "Decomposition of Urea into NH3 for the SCR Process", Ind.Eng.-Chem.Res, 43 (1), 4856-4863.

http://dx.doi.org/10.1021/ie034052j
Koebel, M., Elsener, M., Kleemann, M., 2000, “Urea-SCR: a Promising Technique to Reduce $\mathrm{NO}_{\mathrm{x}}$ Emissions from Automotive Diesel Engines", Catalysis Today, 59, 335-345.

http://dx.doi.org/10.1016/S0920-5861(00)00299-6

Kontin, S., Höfler, A., Koch, R., Bauer, H., 2010, "Heat and Mass Transfer Accompanied by Crystallisation of Single Particles Containing Urea-Water-Solution", 23rd Annual Conference on Liquid Atomization and Spray Systems, Brno, Czech Republic. 